\title{
Implementação do Módulo Web Baseado na Arquitetura Cliente-Servidor para o Aplicativo Móvel Educacional e Open-Source Sem!o
}

\author{
Uma Ferramenta Educacional de Apoio ao Ensino-Aprendizagem da Semiologia Médica
}

\author{
Gabriele Bueno de Oliveira, Jeziel Mateus de \\ Abreu \\ Universidade Federal da Integração Latino-Americana \\ UNILA \\ Foz do Iguaçu, PR, Brasil \\ \{gb.oliveira.2017, jmd.abreu.2016\}@aluno.unila.edu.br
}

\author{
Joylan Nunes Maciel, Marcelo Nepomoceno Kapp \\ Universidade Federal da Integração Latino-Americana \\ UNILA \\ Foz do Iguaçu, PR, Brasil \\ \{joylan.maciel, marcelo.kapp\}@unila.edu.br
}

\begin{abstract}
Resumo-O uso das tecnologias de informação na saúde tem promovido contribuições positivas no ensino-aprendizagem da medicina. Este trabalho apresenta o processo de desenvolvimento de um módulo web e sua integração ao aplicativo móvel educacional e open-source Sem!o, o qual é utilizado no processo de ensino-aprendizagem da semiologia médica. O módulo web está em fase de implementação e emprega a arquitetura Cliente-Servidor, o padrão arquitetural REST, as ferramentas da plataforma Google Android e o modelo de desenvolvimento de software do Processo Unificado. A construção do módulo web permitirá o uso do aplicativo Sem!o por diversos usuários simultaneamente, via Internet, agregando características de interatividade e estimulando um melhor aprendizado dos termos médicos para os estudantes de medicina. Isto contribuirá positivamente na qualificação dos futuros profissionais médicos, pois o correto significado dos termos médicos promove diagnósticos mais precisos e efetivos para a sociedade. As características de flexibilidade de acesso e atualização da base de dados do aplicativo Sem!o são diferenciadas e o tornam aplicável em qualquer domínio do conhecimento na educação.
\end{abstract}

Keywords: Ensino-Aprendizagem; Android; REST; Aplicativo Web; Semiologia Médica.

\section{INTRODUÇÃO}

A geração de dispositivos móveis atuais permite que os usuários realizem diversas tarefas simultaneamente, antes realizadas por computadores desktop conectados ou não à Internet. Podem-se destacar como principais características da computação móvel a mobilidade e a flexibilidade que engloba também portabilidade, usabilidade, popularidade e conectividade desses dispositivos [1].

O uso de técnicas computacionais como instrumento de apoio à medicina tem gerado contribuições importantes e positivas, entre as quais, intercâmbio de informações médicas, aprimoramento de serviços e diagnósticos, a eficiência na tomada de decisão quanto à escolha da terapêutica realizada, aperfeiçoamento da educação médica, inclusive à distância [2,3,4] .

Sob a perspectiva do ensino-aprendizagem da medicina, o uso das tecnologias de informação tem promovido grandes contribuições positivas na prática do ensino e da pesquisa $[5,6,7,8]$. Mais especificamente sobre o aprendizado e conhecimento de termos médicos, e conceitos relacionados a estes termos, diversas soluções computacionais estão disponíveis para acesso e utilização, tais como: o Sheppard Software [9] que consiste em um jogo para o aprendizado e melhora do vocabulário médico em inglês; o site Sporcle [10] que permite a criação de jogos de perguntas sobre termos médicos em inglês; o aplicativo (app) Medical Terminology Quiz [11] e Learn Medical Teraminology [12], os quais permitem a consulta e aprendizado de termos médicos em inglês; os aplicativos Terminologia Médica Free [13] e Dicionário de Saúde Offline [14] que permitem a consulta de termos médicos em português, tal como um dicionário ou glossário, dentre outras aplicações

Ainda nesse cenário, destacam-se dois aplicativos: o SemioQuiz [15] que fornece um Quiz sobre a semiologia médica e o Kahoot [16], um app interativo que permite ao docente cadastrar questões individuais e realizar campeonatos de quiz em sala de aula, em qualquer idioma. Alguns dos apps listados anteriormente permitem realizar jogos de pergunta e respostas (quizzes) e promovem maior interatividade do usuário durante o processo de aprendizagem. Todavia, a maioria destes aplicativos são para a língua inglesa ou não são livres, restringindo o acesso gratuito a todas as funcionalidades e, principalmente, ao banco de dados do app. No caso do Kahoot, apesar da interatividade promovida e de aceitar diversos idiomas, falta flexibilidade durante o cadastramento das questões que deve ser feito de modo individual e manual, exigindo tempo e dedicação dos docentes e gestores. Além disso, o quiz em grupo do Kahoot funciona somente online via Internet.

Nesse sentido, este trabalho tem o objetivo geral de projetar e desenvolver o módulo de funcionamento web, baseado na arquitetura Cliente-Servidor [17], para o aplicativo móvel educacional Sem!o [18], um software livre que está sendo desenvolvido pelo Núcleo de Informática em Ciências da Saúde ${ }^{1}$ da Universidade Federal da Integração Latino-Americana (UNILA). Aplicado inicialmente na educação médica, os principais diferenciais do Sem!o consistem na flexibilidade de gestão e atualização do banco de dados de questões [19], na interatividade promovida com sua utilização e possibilidade de expansão e aplicação em outros domínios do conhecimento. Isto pode contribuir para melhorar o processo de ensino-aprendizagem educacional em qualquer área da educação. Desse modo, os objetivos

1 Informações adicionais disponíveis em $<$ http://nics.tk $>$. 
específicos consistem em: 1) avaliar as tecnologias e métodos de desenvolvimento de aplicativos móveis em Android atuais baseadas na arquitetura Cliente-Servidor; 2) implementar o servidor (back-end) para tratamento das requisições e respostas para o banco de dados; e 3) desenvolver o cliente (front-end) para o sistema operacional móvel Google Android, permitindo ao usuário manipular dos dados por meio da interface gráfica interativa. Portanto, este trabalho está organizado do seguinte modo: a seção II apresenta as ferramentas, materiais e métodos empregados no desenvolvimento, a seção III exibe os resultados alcançados até o momento, e a seção IV as considerações finais e trabalhos futuros.

\section{MATERIAIS E MÉTODO}

Considerando os objetivos definidos na Seção I, um estudo bibliográfico foi realizado acerca das tecnologias, ferramentas e metodologias de desenvolvimento opensource, as quais são detalhadas a seguir.

\section{A. Tecnologias e Ferramentas}

a) Linguagem PHP: A linguagem de programação PHP surgiu em 1994 e desde então vem sendo aprimorada e utilizada para desenvolvimento web. Possui vantagem em relação a outras linguagens que têm o mesmo propósito por ser multiplataforma, dinâmica e gratuita. Além disso, é uma linguagem de execução robusta, sólida e estável em relação a implementação e quantidade de recursos disponíveis [20]. Os códigos PHP são executados no servidor (back-end), cuja função é responder as requisições, executar scripts de processamento e enviar os resultados para o cliente. Portanto, a linguagem PHP foi empregada na implementação back-end do app proposto.

b) Servidor de banco de dados MySQL: A principal função dos bancos de dados é o armazenamento das informações manipuladas no app. Geralmente utiliza um modelo de representação relacional dos dados, ou seja, um grupo de registros formando uma ou várias tabelas que facilitam a organização e a comunicação entre os dados em documentos ou softwares [21]. A gestão do banco de dados é realizada por Sistemas de Gerenciamento de Banco de Dados (SGBD), responsáveis por disponibilizar uma interface para que programas e usuários externos acessem o banco de dados, realizem backups, consultas e manipulação com controle de acesso à informações. Neste trabalho utilizou-se o SGBD MySQL por ser gratuito, estável e atender aos requisitos do app proposto.

c) Servidor de Aplicação Apache e XAMPP ${ }^{2}$ : Empregou-se neste trabalho o Apache como servidor de aplicação, sendo que este é o servidor web mais utilizado no mundo e trabalha com uma grande variedade de ambientes e plataformas [17]. É responsável pela gestão do protocolo Hypertext Transfer Protocol (HTTP) e documentos Hypertext Markup Language (HTML), disponibilizando arquivos no formato de hipertexto e realizando a conexão entre os navegadores (web browsers) do usuário e o servidor. Além disso, na construção da solução utilizou-se a ferramenta de

2 Mais detalhes disponíveis em < https://www.apachefriends.org $>$. desenvolvimento open-source $\mathrm{XAMPP}^{3}$, que agrega o banco de dados, o servidor de aplicação Apache e o ambiente de desenvolvimento necessário ao projeto. O XAMPP é gratuito e de fácil instalação, viabiliza a construção de aplicações web provendo uma comunicação efetiva entre o cliente e servidor.

d) Padrão arquitetural REST e Framework SLIM: Aplicou-se o modelo arquitetural Representational State Transfer (REST) proposto em [22], cujo padrão é baseado no conceito de recursos e emprega os métodos GET, PUT, POST, DELETE e etc, do protocolo HTTP para a realização e tratamento das requisições e respostas em um sistema web Cliente-Servidor. Tais métodos não manipulam os recursos, mas a representação de seus estados [22]. Os serviços que seguem o padrão arquitetural REST são denominados RESTful e provêem maior facilidade para o desenvolvimento de aplicativos móveis, uma vez que a praticidade é incorporada por conta da demanda de restrições que os equipamentos impõem [23]. A implementação REST foi elaborada por meio do Slim Framework ${ }^{4}$, cuja função consiste em facilitar a criação de rotas e Uniform Resource Locator (URL), atuando principalmente na abstração dos controladores presentes no servidor. Estes controladores recebem as requisições HTTP dos clientes, as processam de acordo com a rota (URL) utilizada e retornam as respostas [17].

e) Linguagem Java e Plataforma Android Studio: Java é uma linguagem de programação orientada a objetos e utilizada em uma vasta gama de plataformas e ambientes heterogêneos. Provê compatibilidade, segurança e foi a primeira linguagem nativa utilizada no desenvolvimento de aplicativos para Android [24]. A plataforma oficial da Google para desenvolvimento de app nativos Android é o ambiente de programação Android Studio ${ }^{5}$, que permite empregar a linguagem Java e a edição inteligente de códigos contendo diversos recursos visuais que facilitam a construção da interface gráfica do usuário [25].

f) Biblioteca Retrofit: É uma biblioteca de recursos open-source que intermedia a comunicação web entre back-end e front-end [26], transformando a comunicação HTTP em uma interface de programação Java. Utiliza anotações HTTP em Java que são responsáveis por fornecer o método de requisição e sua respectiva URL. Também permite serializar os dados por meio de um conversor denominado GSON, trabalhando com chamadas (calls) que fazem requisições (síncronas ou assíncronas) ao servidor back-end [26].

\section{B. Método de Desenvolvimento}

O módulo web do Sem!o foi projetado com base na arquitetura Cliente-Servidor [17], em que o servidor trata e responde as requisições, via protocolo HTTP, que são realizadas pelos clientes, neste caso app Android. Esta

3 Detalhes disponíveis em <

https://www.apachefriends.org/blog/news-article-61070.html >

4 Detalhes disponíveis em < http://www.slimframework.com $>$.

5 Detalhes disponíveis em $<$ https://developer.android.com/docs $>$. 
arquitetura simplifica o desenvolvimento e a manutenção, sendo seus componentes detalhados a seguir:

a) Servidor (back-end): O servidor desenvolvido em PHP realiza a comunicação entre o banco de dados e o tratamento de requisições HTTP, executando manipulações de inserção, consultas, edições e exclusões no banco de dados com base nos métodos HTTP (POST, GET, DELETE, etc). Toda requisição válida recebida pelo servidor gera uma resposta ao cliente solicitante. Após a consulta ao banco de dados o servidor retorna uma resposta de sucesso ou falha. Essas respostas, quando necessário, contêm dados encapsulados no formato JSON.

$\mathrm{Na}$ Figura 1 é demonstrado o código PHP de tratamento do servidor para tratar uma requisição de consulta de Usuário e seus atributos. Observa-se o método HTTP GET utilizado para a URL (end-point) /consultarUsuarios, a chamada do serviço de consulta de usuários no banco de dados $\$ d b$->consultarUsuarios() e a resposta enviada para o cliente no formato JSON json_encode(\$response_data).

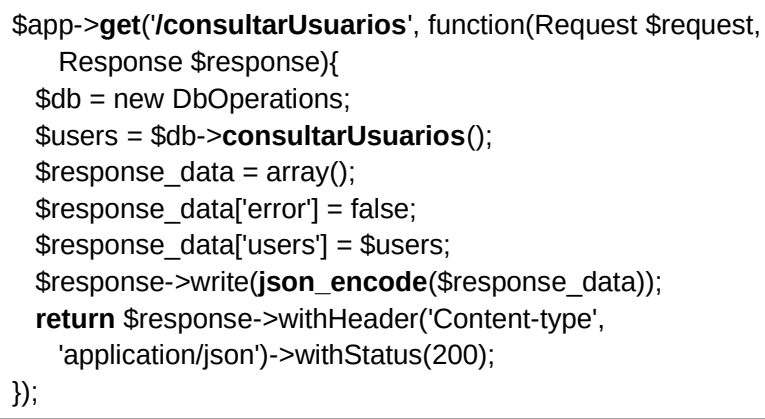

Figura 1. Tratamento de uma requisição no servidor (back-end).

b) Cliente (front-end): A aplicação cliente foi desenvolvida para o sistema operacional Google Android, por meio da linguagem Java e biblioteca Retrofit, cuja classe essencial para funcionamento da comunicação de dados entre o cliente e servidor é relativa ao objeto Service e sua respectiva interface. A Figura 2 representa a realização de uma requisição de cadastro de usuário pelo cliente, na qual observa-se a requisição com o método POST e seu end-point, a chamada do serviço ao servidor “cadastroUsuario” e o atributos @Field do usuário a ser cadastrado.

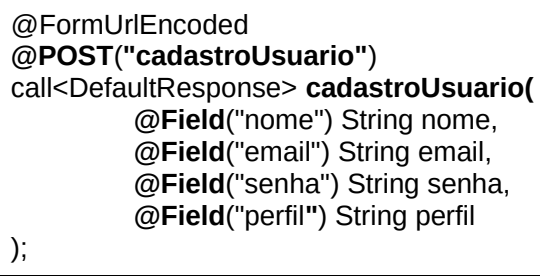

Figura 2. Requisição do cliente (front-end) com a biblioteca Retrofit.

A Figura 3 apresenta o tratamento da resposta recebida no cliente para a requisição de cadastro de usuário da Figura 2. Observa-se que o objeto da chamada call trata os dados enviado pelo servidor ao cliente, ou seja, a resposta relativa ao serviço cadastroUsuario(...). Essa resposta tratada no método OnResponse(...) pode ser de usuário criado (código 201) ou usuário existente (código 422), ou ainda alguma falha tratada por meio do método onFailure(...).

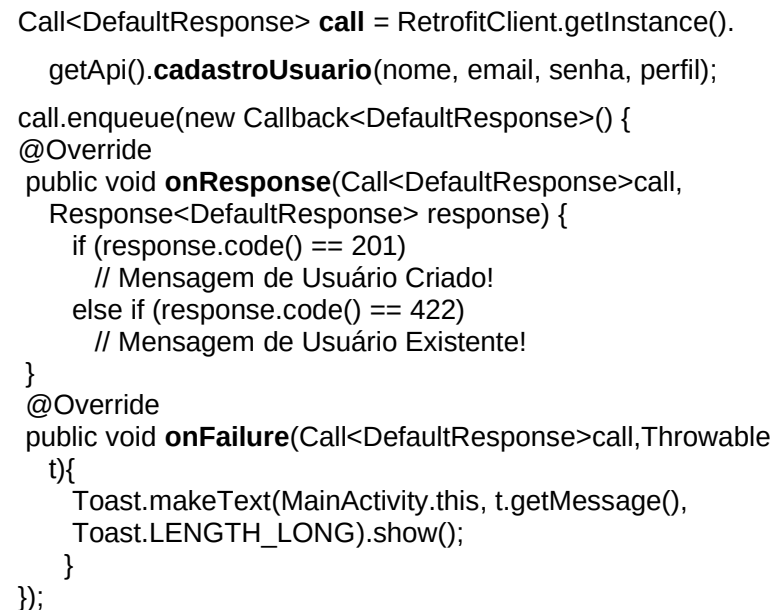

Figura 3. Tratamento de uma resposta no cliente (front-end) por meio da biblioteca Retrofit.

De modo similar as operações de criação, listagem, alteração e remoção (CRUD) foram desenvolvidas para os os usuários do sistema por meio do módulo web. O desenvolvimento empregou a biblioteca Retrofit e seguiu o modelo de processo de software Processo Unificado [27].

\section{RESULTADOS}

As principais funcionalidades não web e existentes no aplicativo Sem!o estão representadas no Diagrama de Casos de Uso [27] exibido na Figura 4. Até o momento, a integração ao módulo web foi desenvolvida, está testada e funcional para os casos de uso Gerenciar Usuários e Gerenciar Categorias, conforme identificado na Figura 4.

Figura 4: Diagrama de Casos de Uso do Sem!o. Adaptado de [28].

Na Figura 5 são apresentadas exemplos de telas referentes a interface do usuário no cliente do aplicativo Sem!o. Na esquerda é exibida a tela inicial do sistema para login dos usuários no app, no centro a interface de listagem dos usuários já cadastrados no servidor da aplicação, e à direita é apresentada a tela de edição das informações de usuários, as quais são posteriormente persistidas no servidor. 
Figura 5. Interfaces do módulo web (front-end) do app Sem!o: tela de login (esquerda), listagem (centro) e edição de usuário (direita).

\section{CONSIDERAÇÕES FINAIS E TRABALHOS FUTUROS}

Este trabalho apresentou o processo de desenvolvimento e integração de um módulo web, baseado na arquitetura Cliente-Servidor e modelo arquitetural REST, ao app móvel, educacional e open-source Sem!o. A implementação do módulo web já foi realizada para os casos de uso Gerenciar Usuários e Categorias. Os resultados demonstram a viabilidade dos materiais e métodos empregados, validando a solução adotada.

Desenvolver todas as funcionalidades do Sem!io no modelo web possibilitará o uso simultâneo do app por vários usuários, permitindo o acesso a competições online de quizzes e aumentando a interatividade entre os participantes. Isto promoverá o estímulo acadêmico quanto ao aprendizado de termos médicos, de forma lúdica e interativa, cenário facilitado pela ampla acessibilidade estudantes ao uso de smartphones com o Android.

Por fim, destaca-se a liberdade de atualização e modificação das informações na base de dados do Sem!o, a qual pode ser realizada em "lote” (vários registros de uma vez). Esta característica amplia as possibilidades de utilização, tornando-o flexível e expansível e aplicável em qualquer tema ou área do conhecimento para o ensinoaprendizagem educacional. Trabalhos futuros consistem finalizar o desenvolvimento dos casos de uso na versão web, testá-los, validá-los e incluir melhorias nas funcionalidades do app Sem!o.

\section{REFERÊNCIAS}

[1] Lee U., Han K., Cho H., Chung K., Hong H., Lee S., Noh Y., Park S., Carroll J. M.. Intelligent positive computing with mobile, wearable, and IoT devices: Literature review and research directions. Ad Hoc Networks, Volume 83, 2019, 8-24, ISSN 15708705, 2019.

[2] Urtiga, K. S.; Louzada, L. A. Telemedicina: Uma visão geral da arte. Congresso Brasileiro de Informática Médica, 2004.

[3] Costello E, Corcoran, M, Barnett, J.S, Birkmeier, M. Information and Communication Technology To Facilitate Learning for Health Professions. Online Learning, v.18, n.4, p.1-17, 2014.

[4] Martin Florence, Ertzberger Jeffrey, Here and now mobile learning: An experimental study on the use of mobile technology, Computers \& Education, Volume 68, 76-85, ISSN 03601315,2013

[5] Maciel, J. N. Protótipo de conferência multimídia e transmissão de dados de experimentos médicos em tempo real pela Web. (Monografia). Universidade Estadual do Oeste do Paraná. 2005.
[6] Jin, J.; Bridges, S. M. Educational technologies in problem-based learning in health sciences education: A systematic review. Journal of Medical Internet Research, v. 16, n. 12, p. 1-13, 2014.

[7] Mccoy, L. et al., Developing Technology-Enhanced Active Learning for Medical Education: Challenges, Solutions, and Future Directions. The Journal of the American Osteopathic Association J Am Osteopath Assoc, v. 115115, n. 44, p. 202-211, 2015.

[8] Free, C. et al., The Effectiveness of Mobile-Health Technologies to Improve Health Care Service Delivery Processes: A Systematic Review and Meta-Analysis. PLoS Medicine, v. 10, n. 1, 2013.

[9] Sheppard, Sheppard Software: we make learn fun. Disponível em $<$ http://www.sheppardsoftware.com/web_games_vocab_med .htm $>$. Acesso set. 2017.

[10] Sporcle. Can you pick the correct medical term starting with 'R'. Sporcle Inc. Disponível em <https://www.sporcle.com /games/Bellelady/medical-terms-letter-r>. Acesso set. 2017.

[11] Mcgrath, John. Medical Terminology Quiz. Disponível em < https://play.google.com/store/apps/details? id=appinventor.ai_dot_dox.TheMTQuiz>.>. Acesso set. 2017.

[12] Mcgrath, John. Learn Medical Terminology. Disponível em $<$ https://play.google.com/store/apps/details?

id=appinventor.ai_dot_dox.InterActiveMT $>$. Acesso set. 2017.

[13] MGS. Terminologia médica (Free). Medical Group Software. Disponível em < https://play.google.com/store/apps/details? id=com.medicalgroupsoft.medical.directorymedtermsmultilang.fre e>. Acesso set. 2017.

[14] Ufostudio, Dicionário da Saúde Offline. Disponível em < https://play.google.com/store/apps/details?id=com.ufo.disease >. Acesso set. 2017.

[15] Lasemi. Aplicativo: SemioQuiz. Disponível em $<$ https://play.google.com/store/apps/details? id=com.LASEMI.SemioQuiz\&hl=pt_BR>. Liga LASEMI-UFBA. Acesso set. 2018. 2018.

[16] Kahoot. Kahoot!. Disponível em <https://kahoot.com/>. Acesso set. 2019.

[17] Alves, W. P. Projetos de Sistemas Web. Conceitos, Estruturas, Criação de Banco de Dados e Ferramentas de Desenvolvimento. Editora Érica. $1^{\mathrm{a}}$ ed, 2015. ISBN-13: 978-8536510859.

[18] Mendoza A. D., Wolfgram E., Oliveira S. P., Maciel, J. N., Desenvolvimento do Protótipo de um Aplicativo para o Estudo da Semiologia Médica. Resumo - Anais do XVI Congresso Brasileiro de Informática em Saúde - CBIS 2018. Fortaleza-CE. 2018.

[19] Wolfgram E., Mendoza A. D., Oliveira S. P., Maciel, J. N., Definição de Requisitos e Construção do Banco de Dados de um Protótipo Educacional de Aplicativo Móvel para o Estudo da Semiologia Médica. Resumo - Anais do XVI Congresso Brasileiro de Informática em Saúde - CBIS 2018. Fortaleza-CE. 2018.

[20] Powers D. PHP: A Quick Reference. In: PHP 7 Solutions. Apress, Berkeley, CA. 2019.

[21] Silberchatz A.; Korth H. F.; Sudarshan S. Sistema de Banco de Dados. Editora Campus. 6a Ed. ISBN 9788535245356. 2012.

[22] Fielding, R. T.. Architectural Styles and the Design of NetworkBased Software Architectures. Ph.D. Dissertation. University of California, Irvine. 2000

[23] Halili, F.; Ramadani, E. Web Services: A Comparison of Soap and Rest Services. Modern Applied Science; Vol. 12, No. 3; 2018. ISSN 1913-1844.

[24] Deitel P., Deitel H., Deitel A. An droid how to Program. Prentice Hall Press, Upper Saddle River, NJ, USA. 2013.

[25] DiMarzio J. F.,Beginning Android $®$ Programming with Android Studio. 4ed., John Wiley \& Sons, ISBN:9781119419334, 2016.

[26] Retrofit. A type-safe HTTP client for Android and Java. Disponível em <https://square.github.io/retrofit>. Acesso: 17 jul. 2019.

[27] Pressman R. S. Engenharia de Software - Uma Abordagem Profissional. Editora Bookman. $7^{\mathrm{a}}$ Ed. ISBN: 9788563308337. 2011.

[28] Wolfgram, E. ; Mendoza, A. D. ; Maciel, J. N. ; Oliveira, S. P. . Protótipo Computacional de Apoio à Educação Médica: Análise de Requisitos e Projeto do Banco de Dados. Anais do VII EICTIUNILA, 2018. 